\title{
Head-Tail Instability Caused by Electron Clouds in Positron Storage Rings
}

\author{
K. Ohmi ${ }^{1}$ and F. Zimmermann ${ }^{2}$ \\ ${ }^{1}$ KEK, Oho, Tsukuba, Ibaraki 305, Japan \\ ${ }^{2}$ CERN CH-1211, Geneva 23, Switzerland
}

(Received 12 May 2000)

\begin{abstract}
In positron or proton storage rings with many closely spaced bunches, an electron cloud can build up in the vacuum chamber due to photoemission or secondary emission. We discuss the possibility of a single-bunch two-stream instability driven by this electron cloud. Depending on the strength of the beam-electron interaction, the chromaticity and the synchrotron oscillation frequency, this instability either resembles a linac beam breakup or a head-tail instability. We present computer simulations of the instabilities, and compare the simulation results with analytical estimates.
\end{abstract}

PACS numbers: 29.27.Bd, 29.20.Dh

The luminosity of $B$ factories or Large Hadron Colliders and the resulting physics discovery potential depend on the bunch spacing and the beam sizes. However, narrow spacing, which nominally increases luminosity, can lead to an instability that blows up the beam size and limits achievable luminosity.

For linear accelerators, a single-bunch instability of a positron bunch due to electrons created by ionization of the residual gas has been discussed [1], where a coherent oscillation of both electrons and positrons grows from any small initial perturbation of the bunch distribution, e.g., from the statistical fluctuations due to the finite number of beam particles. This instability can be considered as a two stream instability of the same type as studied in plasma physics.

A similar two-stream instability may occur in positron storage rings due to interaction with electrons generated by photoemission and secondary emission [2]. Such instability could be fast, since in the new generation of storage rings, operating with many closely spaced bunches, the density of the electron cloud which accumulates in the vacuum chamber can become large. Without synchrotron motion, this two-stream instability resembles the classical beam breakup (BBU), and manifests itself in a coherent dipole oscillation along the bunch. In a storage ring the BBU appears as either strong or regular head-tail instability, due to synchrotron oscillation and, possibly, chromaticity. In this Letter, we study the BBU and the head-tail instability caused by the electron cloud using a computer simulation.

The photoelectrons produced by synchrotron radiation in positron storage rings may also cause a multibunch dipole-mode instability [3]. This multibunch instability is different from the instability discussed in this Letter, which is a single-bunch phenomenon caused by the photoelectron cloud. Although a single-bunch effect, the latter will, however, occur only in multibunch operation, since the electron cloud is built up from synchrotron radiation emitted by the preceding bunches. The head-tail mode of the singlebunch instability will be observed as a beam-size blowup.

As a concrete example, we study the single-bunch photoelectron instability for the Low Energy Ring of the
KEK- $B$ factory (KEKB-LER), which is a $3.5 \mathrm{GeV}$ positron storage ring with the circumference $L=3016 \mathrm{~m} \mathrm{[4].}$ At the beginning of the year 2000, the LER was operated with a beam current of $600 \mathrm{~mA}$ stored in 1000 bunches at 8 ns spacing. A blowup of the vertical beam size has been observed already early on during LER commissioning [5]. This blowup is not accompanied by any coherent beam motion, which is suppressed by transverse feedback and chromaticity, and the blowup is seen only in multibunch operation with a narrow bunch spacing. The single-bunch two-stream instability provides a plausible explanation of the observed beam blowup.

We, first, discuss the density of electron cloud near the beam, then describe the simulation model for the motion of a positron bunch passing through an electron cloud, next present simulation results of beam breakup and head-tail instability, and, finally, compare the simulated instability rise times with analytical estimates.

Photoelectrons produced by synchrotron radiation are the major source of electrons in the vacuum chamber of the KEKB-LER. At the LER, a positron emits about 450 photons in one revolution, or an entire positron bunch emits $5 \times$ $10^{9}$ photons per meter, where the bunch population is $N_{b}=$ $3.3 \times 10^{10}$. Assuming a photoelectron yield of 0.1 , the number of photoelectrons is estimated to be $5 \times 10^{8} / \mathrm{m}$.

We obtain the quasistationary electron-cloud density, $\rho$, from a computer simulation which models the motion of photoelectrons in the electric field of the positron beam during successive bunch passages. This calculation is performed following the same procedure as was used for studying the multibunch electron-cloud instability in Ref. [3]. The initial energy distribution of the electrons is assumed to be a truncated Gaussian with peak energy $5 \mathrm{eV}$ and standard deviation $10 \mathrm{eV}$, restricted to positive values. Most electrons are created at the side wall of the chamber where the primary synchrotron radiation impinges, but a significant portion $(30 \%)$ is created uniformly around the chamber wall to represent the contribution from reflected photons. Whenever electrons are lost to the wall, secondary electrons are emitted. Their number depends on the assumed secondary emission yield, the primary energy, and the angle of 
incidence. The simulation also takes into account the space charge force between electrons [6].

Figure 1(a) shows electron-cloud densities obtained by the simulation. Both average and local densities (at the beam-pipe center) are depicted. The number of photoelectrons increases at each bunch passage and, finally, saturates when a quasistationary value is reached representing a dynamic equilibrium between creation and absorption. After a few bunch passages the central electron density exceeds $10^{12} \mathrm{~m}^{-3}$. An average electron density of $6 \times 10^{11} \mathrm{~m}^{-3}$ corresponds to a line density $5 \times 10^{9} \mathrm{~m}^{-1}$. Saturation occurs when the electron line density becomes comparable to the line density of the beam $\left(1.4 \times 10^{10} \mathrm{~m}^{-1}\right)$, in which case the time-averaged electric field at the chamber wall vanishes. Hence, the neutralization level may be defined as the ratio of the line densities of electrons and beam. Figure 1(b) shows the velocity distribution of electrons close to the bunch.

We have written a second simulation program to study the coupled motion of a single positron bunch and the cloud electrons. Perturbations of the cloud due to the preceding bunches are neglected; that is, in the simulation a positron bunch always interacts with the quasistationary unperturbed electron cloud. The unperturbed electron distribution is taken to be Gaussian, with transverse rms sizes and electron-cloud density as input parameters. For simplicity, the electron cloud is assumed to be localized at a single position of the ring, denoted by $s_{e}$. The cloud density at this location is chosen such that the average electron density over the ring circumference is equal to the actual value. Note that the localization of the electrons ignores the frequency spread of the electron oscillations which would arise from a variation in beta functions and dispersion. If the number of oscillations over the bunch length is small (in our KEKB example it is of the order of one), the detuning due to such frequency spread will be small; see also the analytical expression of the detuning effect for the case of the fast beam-ion instability in Refs. [7,8]. The positron bunch with length $\sigma_{z}$ is sliced into a number of microbunches $\left(N_{p}\right)$ in the longitudinal direction. Each microbunch has a transverse beam size $\left(\sigma_{x}, \sigma_{y}\right)$ determined by emittance and beta function. The interaction between a microbunch ( $i$ th) and the cloud electrons is
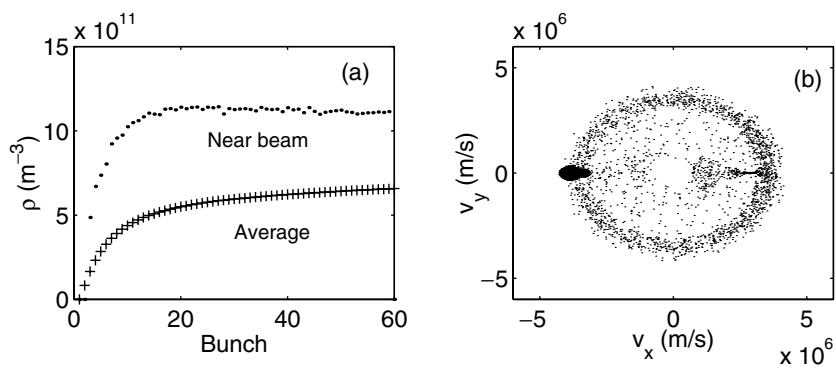

FIG. 1. Density and velocity distributions of electron cloud for $8 \mathrm{~ns}$ bunch spacing. (a),(b) The electron densities and the velocity distributions, respectively. The crosses and dots in (a) refer to the average density and to the local density near the beam. expressed by two equations [9],

$$
\begin{aligned}
\Delta \overline{\boldsymbol{x}}_{p, i}^{\prime} & =-\frac{2 r_{e}}{\gamma} \sum_{a=1}^{N_{e}} \boldsymbol{F}_{G}\left[\overline{\boldsymbol{x}}(s)_{p, i}-\boldsymbol{x}_{e, a} ; \boldsymbol{\sigma}\right], \\
\Delta \dot{\boldsymbol{x}}_{e, a} & =-\frac{2 N_{b} r_{e} c}{N_{p}} \boldsymbol{F}_{G}\left(\boldsymbol{x}_{e, a}-\overline{\boldsymbol{x}}_{p, i} ; \boldsymbol{\sigma}\right),
\end{aligned}
$$

where the force $\boldsymbol{F}_{G}(\boldsymbol{x})$ is expressed by the Bassetti-Erskine formula [10] normalized so that $\boldsymbol{F}_{G} \rightarrow \boldsymbol{x} /|\boldsymbol{x}|^{2}$ as $\boldsymbol{x} \rightarrow \infty$. Equation (2) shows that the electrons oscillate near the beam center with the angular oscillation frequency

$$
\omega_{e}^{2}=\frac{2 \lambda r_{e} c^{2}}{\sigma_{y}\left(\sigma_{x}+\sigma_{y}\right)},
$$

where $\lambda=N_{b} /\left(2 \sigma_{z}\right)$ denotes the line density of the positron bunch. For KEKB $\left(\sigma_{x}=420 \mu \mathrm{m}, \sigma_{y}=60 \mu \mathrm{m}\right.$, $\left.\sigma_{z}=4 \mathrm{~mm}\right), \omega_{e}=2 \pi \times 45 \mathrm{GHz}$.

The simulation is performed by successively solving the motion of positron microbunches and macroelectrons using Eqs. (1) and (2), respectively. The simulation is quite similar to that of ion instabilities [9]. A series of microbunches corresponds to a bunch train in the ion problem. Main differences are the narrow longitudinal spacing of the positron microbunches, the variation of their longitudinal positions due to synchrotron motion, and the higher oscillation frequency in the bunch potential.

The electron cloud is represented by macroparticles. The distribution of these macroparticles is assumed to be Gaussian with a size of $\sim 3 \mathrm{~mm}$, which is 8 or 50 times larger than the horizontal and vertical rms beam sizes, respectively. Usually the initial macroelectron velocities are set to zero, but they can be varied in order to study the dependence on this parameter. We represent a positron bunch by about 1000 microbunches and the electron cloud by 10000 macroparticles. An unperturbed fresh electron distribution is generated on each revolution.

Figure 2 shows the transverse amplitudes of microbunches distributed over the longitudinal phase space, for two cases. For easy visualization, we use a multiple airbag model for the longitudinal microbunch distribution, in which the microbunches are initially distributed on concentric circles in the longitudinal phase space, characterized by the position $z$ and the relative energy deviation $\Delta p / p$. Figure 2(a) depicts the deformation of the positron bunch without synchrotron oscillations. Clearly visible is a

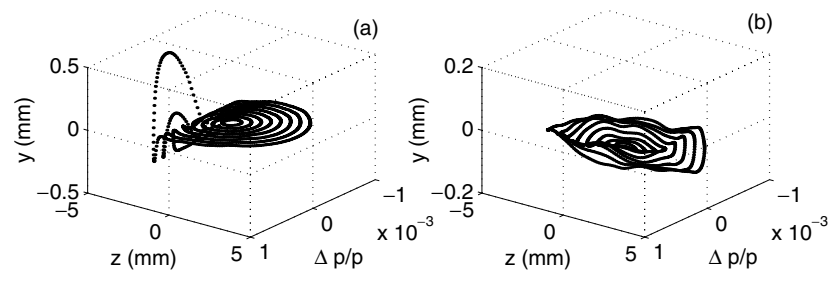

FIG. 2. Bunch shape deformation due to the interaction with electron cloud. The positions of the microbunches are plotted after 100 turns for a cloud density of $1 \times 10^{12} \mathrm{~m}^{-3}$. (a),(b) Cases without and with synchrotron oscillations, respectively. The synchrotron tune for case (b) is $Q_{s}=0.015$. 
transverse oscillation along the bunch, the frequency of which is consistent with Eq. (3). The figure is reminiscent of the BBU observed in linear accelerators. Including also the synchrotron oscillation, the beam breakup changes its appearance and now resembles a head-tail instability. Figure 2(b) shows the bunch shape deformation with synchrotron oscillations for a synchrotron tune $Q_{s}=0.015$. We find a correlation of transverse amplitude and longitudinal phase space position, which is characteristic of head-tail motion. Note that the magnitude of the oscillation amplitudes is reduced by the synchrotron motion.

The conventional head-tail effect caused by a short range wake force [11] results in damping of the dipole $(l=0)$ mode and a growth of higher order $(l \geq 1)$ head-tail modes for positive chromaticity, and roughly the opposite behavior for negative chromaticity. For zero chromaticity, the beam is stable at low current. Above a certain current threshold some head-tail modes are coupled and strongly excited. This is called the strong head-tail instability. The simulation reveals exactly the same dependence for the electron-cloud wake.

Excitation of the higher order head-tail mode is observed as a blowup of the vertical size of the positron bunch. We quantify this blowup by computing the root mean square of all microbunch amplitudes, $\sqrt{\left\langle y_{p}^{2}\right\rangle}$. Figure 3 shows the growth of this quantity for the BBU mode, without synchrotron motion, in 3(a), and for the head-tail mode oscillation with a synchrotron tune $Q_{s}=0.015$, in 3(b) and 3(c). The latter two examples were calculated for $Q_{x, y}^{\prime}=(4,8)$ and $(0,0)$, respectively. We now use a Gaussian distribution for microbunches in the longitudinal phase space. The three curves refer to different cloud densities. The growth rate is of the order $\sim 0.1 \mathrm{~ms}$ for the BBU mode, as illustrated in 3(a). The behavior with synchrotron motion is different for positive and for zero chromaticity. At $Q_{x, y}^{\prime}=$ $(4,8)$ the growth time is about $1 \mathrm{~ms}$ for the two lower electron densities, whereas at $Q_{x, y}^{\prime}=(0,0)$ it is much slower. For the highest density, $\rho=1 \times 10^{12} \mathrm{~m}^{-3}$, the growth at both chromaticities is about the same and extremely fast, with a rise time of the order of $0.2 \mathrm{~ms}$. Our interpretation is that there is a threshold value for the electron-cloud density above which we observe the strong head-tail instability.

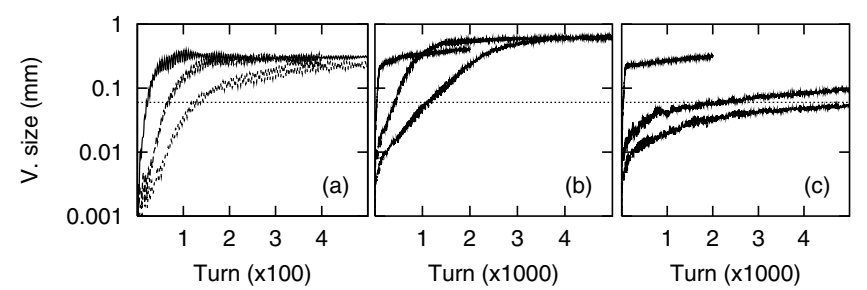

FIG. 3. Growth of the vertical rms amplitude of the microbunches. The three curves are results for electron-cloud densities of $2 \times 10^{11}, 4 \times 10^{11}$, and $1 \times 10^{12} \mathrm{~m}^{-3}$. The growth is faster for higher density. (a) Obtained without synchrotron oscillations. (b),(c) Chromaticities $Q_{x, y}^{\prime}$ of $(4,8)$ and $(0,0)$, respectively, with synchrotron motion and $Q_{s}=0.015$. The dashed lines indicate the natural beam size.
All simulated growth times are much shorter than the radiation damping time $(40 \mathrm{~ms})$ except for the two cases of zero chromaticity and lower cloud density. The growth tends to slow down for large amplitudes at several $\sigma_{y}$. We attribute this to the nonlinearity of the forces between beam and electrons, which will also lead to filamentation and emittance growth.

Our picture of the two-stream instability is that the cloud electrons oscillate incoherently at first, but, gradually, they and the positron bunch (the microbunches in the simulation) develop a coherent oscillation due to their interaction. After the bunch passage the coherence of the electrons is lost, and on the next revolution the further distorted positron bunch impresses an enhanced coherent motion on the newly formed electron cloud, which in turn increases the oscillation along the bunch.

The force from the electron cloud may be represented by an effective short range wake field with a characteristic frequency as in Eq. (3). The strength of the wake force can be obtained by the same method as in Ref. [3]. The order of magnitude of the wake force may also be estimated analytically. For example, considering a flat beam with $\sigma_{x} \gg \sigma_{y}$, we decompose the electron cloud into infinitely thin vertical slices, each producing the same vertical electric field, and study a two-particle model with a charge of $N_{b} e / 2$ for both head and tail. We assume that the head particle has a finite length $l_{\text {head }} \approx \sqrt{2 \pi} \sigma_{z} / 2$, and a uniform charge distribution. The tail particle is considered to be pointlike and to follow immediately after the head. Head and tail are vertically displaced with respect to each other by a small offset $\Delta y \ll \sigma_{y}$. From the resulting force on the tail we can then estimate the effective wake field. Electrons near the beam are attracted by the field of the head and perform linear or nonlinear oscillations during its passage. Because of the relative displacement of head and tail, these oscillations induce a net electron transfer from below to above the vertical position of the trailing particle. The electron charge transfer is maximum if $\omega_{e} l_{\text {head }}$ is equal to an odd multiple of $\pi / 2$, reflecting the effect of linearly oscillating electrons within about $\pm 2 \sigma_{y}$ from the beam. At intermediate times, the net charge transfer amounts to the number of electrons which originally occupy a vertical stripe of thickness $\sim 2 \Delta y$, i.e., about twice the displacement.

In this two-particle model, the integrated wake field per revolution experienced by the tail of the bunch is of the order

$$
W_{0} \approx 8 \pi \rho L / N_{b} .
$$

On each turn the tail particle experiences a deflection of

$$
\Delta y_{\text {tail }}^{\prime}=\frac{r_{e} W_{0} N_{b}}{2 \gamma}\left(y_{\text {head }}-y_{\text {tail }}\right),
$$

where $y^{\prime}$ denotes the vertical slope of the trajectory. This estimate is valid if the distance between head and tail is large compared with $\sigma_{x} \sigma_{y} /\left(N_{b} r_{e}\right)$, where $r_{e}$ denotes the classical electron radius. This is usually the case. Unlike 
an ordinary wake field, the wake $W_{0}$ decreases inversely with the population of the bunch considered. However, the population of the previous bunches also enters, indirectly, in the value of $\rho$, so that for equal bunch populations there is no dependence on $N_{b}$. Indeed, assuming that the equilibrium density $\rho$ is equal to the average neutralization density $N_{b} /\left(\pi h_{x} h_{y} L_{\text {sep }}\right)$, where $h_{x}$ and $h_{y}$ are the horizontal and vertical chamber half apertures and $L_{\text {sep }}$ the bunch spacing (in meters), our wake estimate can be rewritten as $W_{0} \approx 8 L /\left(h_{x} h_{y} L_{\text {sep }}\right)$, which depends only on geometric quantities.

On the other hand, if the bunch length is short compared with $\sigma_{x} \sigma_{y} /\left(N_{b} r_{e}\right)$, so that only electrons in the linear part of the beam field contribute to the charge transfer, the wake field can be estimated as $W_{0} \approx 4 \pi \rho L r_{e} l_{\text {head }} /\left(\sigma_{x} \sigma_{y}\right)$.

The growth rate for the BBU mode, without synchrotron motion, can be estimated in the two-particle model using the saturated wake field of Eq. (4):

$$
\frac{1}{\tau} \approx \frac{2 \pi \rho r_{e} c\left\langle\beta_{y}\right\rangle}{\gamma} \text {. }
$$

For KEKB parameters $\left(\left\langle\beta_{y}\right\rangle=10 \mathrm{~m}\right)$ the BBU growth time evaluates to about $100 \mu \mathrm{s}$, in good agreement with the simulation of Fig. 3(a). Modifying the theory for the single-bunch instability due to ionization electrons [1] can give an alternative estimate [2].

Inserting our wake field estimate, Eq. (4), into the standard expression for the regular head-tail growth time [11], we can estimate the growth rate of the $l=1$ head-tail mode as

$$
\frac{1}{\tau^{(1)}} \approx \frac{64}{3} \frac{\rho\left\langle\beta_{y}\right\rangle r_{e} \sigma_{z} Q_{y}^{\prime}}{T_{0} \alpha \gamma},
$$

where $\alpha$ is the momentum compaction factor. For $Q_{y}^{\prime}=8$ this equation predicts a growth time of about $0.5 \mathrm{~ms}$, again in reasonable agreement with the simulation.

Finally, we can calculate the threshold of the strong head-tail instability for the two-particle model. Following Ref. [11], the threshold is reached when the parameter $N_{b} r_{e}\left|W_{0}\right| \beta_{y} /\left(8 \gamma Q_{s}\right)$ is equal to 2 . This translates into a threshold value for the electron-cloud density of

$$
\rho_{\mathrm{thr}}=\frac{2 \gamma Q_{s}}{\pi r_{e} L \beta_{y}},
$$

which evaluates to $7 \times 10^{11} \mathrm{~m}^{-3}$, and agrees surprisingly well with the simulated threshold.

We have studied a single-bunch head-tail instability caused by the photoelectron cloud. The instability depends on the electron-cloud density near the beam. For the KEKBLER this density is estimated to be about $\sim 10^{12} \mathrm{~m}^{-3}$. Simulated instability growth times are of the order of $0.1-$ $1 \mathrm{~ms}$ and consistent with analytical estimates. The typical equilibrium density of the electron cloud for present KEKB parameters is close to the threshold of the strong head-tail instability. In operation with bunch trains, the electron cloud is built up along each train and it is cleared by sufficiently large gaps between subsequent trains. The saturation of the oscillation amplitudes at $\sim 10 \sigma_{y}$, found in the simulation, indicates that the beam is not lost, but that the instability will mainly increase the beam size. The beam size blowup should increase along a bunch train, in parallel with the buildup of the electron cloud at the center of the vacuum chamber. In particular, the instability may cause a beam size blowup as observed at KEKB. It could also explain similar observations at PEP-II [12] and at the CERN SPS [13]. The instability can be suppressed by reducing the electron density near the beam. Therefore, coating of the vacuum chamber and weak magnetic fields are potential cures.

In our simulation, the positron bunch was represented by a large number of microbunches with a fixed transverse beam size. In reality the transverse sizes of the slices will vary under the action of the electron cloud, and other incoherent effects may also become important $[14,15]$. Therefore, more realistic studies of this instability should be performed, e.g., via particle-in-cell simulations [16].

The authors thank H. Fukuma, K. Oide, and other members of the KEKB commissioning group for fruitful comments and discussions, and appreciate support by K. Hübner, F. Ruggiero, and F. Takasaki.

[1] T. O. Raubenheimer and F. Zimmermann, Phys. Rev. E 52, 5487 (1995).

[2] F. Zimmermann, Report No. CERN-SL-Note-2000-004 AP, 2000.

[3] K. Ohmi, Phys. Rev. Lett. 75, 1526 (1995); M. Izawa, Y. Sato, and T. Toyomasu, Phys. Rev. Lett. 74, 5044 (1995).

[4] KEK B-Factory Design Report No. KEK 95-7, 1995.

[5] K. Oide et al., in Proceedings of International Workshop on Performance Improvement of Electron-Positron Collider Particle Factories (KEKB Report No. 99-24, 2000).

[6] K. Ohmi, in Proceedings of 14th Advanced ICFA Beam Dynamics Workshop: Beam Dynamics Issues for $e^{+} e^{-}$ Factories, Frascati, Italy (INFN, Frascati, 1997).

[7] A. Chao and M. Tigner, Handbook of Accelerator Physics and Engineering (World Scientific, River Edge, NJ, 1999).

[8] G. V. Stupakov, T. O. Raubenheimer, and F. Zimmermann, Phys. Rev. E 52, 5499 (1995); G. V. Stupakov, Phys. Rev. ST Accel. Beams 3, 019401 (2000).

[9] K. Ohmi, Phys. Rev. E 55, 7550 (1997).

[10] M. Bassetti and G. Erskine, Report No. CERN ISR TH/80-06, 1980.

[11] A. Chao, Physics of Collective Instabilities in High Energy Accelerators (J. Wiley, New York, 1993).

[12] M. Minty (private communication).

[13] W. Höfle, J. M. Jimenez, and G. Arduini, in Proceedings of CERN SL Chamonix X Workshop (Report No. CERN-SL2000-007-DI, 2000).

[14] M. A. Furman and A. A. Zholents, in Proceedings of the 1999 Particle Accelerator Conference (IEEE, Piscataway, NJ, 1999), p. 1794.

[15] K. Oide (private communication).

[16] S. Krishnagopal, Phys. Rev. Lett. 76, 235 (1996); K. Ohmi, KEK Report No. 99-162, 2000. 